\title{
Validación en Chile de la Escala de Sobrecarga del Cuidador de Zarit en sus versiones original y abreviada
}

\author{
Hayo Breinbauer $K^{\mathrm{a}}$, Hugo Vásquez Va, Sebastián Mayanz $\mathrm{S}^{\mathrm{a}}$, \\ Claudia Guerraa, Teresa Millán $\mathrm{K}^{1}$. \\ O riginal and abbreviated Zarit \\ caregiver burden scales. \\ Validation in Chile
}

\begin{abstract}
Background: Six percent of the Chilean population has a disability requiring assistance with daily-living-activities and $69 \%$ of these individuals are cared by direct family members. The latter are at risk of developing caregiver burden. Zarit scales are used to assess the severity of caregiver burden. Aim: To validate the original and abbreviated Zarit scales for caregiver burden. Material and methods: Two groups of interviewers applied the original and abbreviated Zarit scales, along with a single subjective indicator for burden and surveys for depression, to 32 caregivers from an outpatient clinic in Melipilla, Chile. In 22 subjects, the instruments were applied again, four months later. Results: Both Zarit scales showed high correlation with the subjective indicator for burden and with depression ( $r=0.51$ and 0.67 , respectively), supporting its construct validity. The abbreviated scale had a high correlation with the original scale $(\mathrm{r}=0.92)$, supporting its criterion validity. It had a $100 \%$ sensitivity, $77.7 \%$, specificity, $86.6 \%$ positive predictive value and $100 \%$ negative predictive value to discriminate severe caregiver burden, using the original scale as standard. Both instruments showed high internal consistency (Cronbach alpha $=0.84$ and 0.87 , respectively), inter-observer reliability (intraclass correlation coefficient $=0.81$ and 0.86 , respectively) and stability reliability (Kappa test-retest $=0.91$ and 0.93 , respectively). Conclusions: Both original and abbreviated Zarit burden scales are valid to assess caregivers burden in a Chilean context. The abbreviated scale seemed particularly useful for primary care (Rev Méd Chile 2009; 137: 657-65).
\end{abstract}

(Key words: Caregivers; Dependency burden; Validation studies)

Recibido el 1 de agosto, 2008. Aceptado el 7 de enero, 2009.

Estudio sin financiamiento externo.

${ }^{1}$ Departamento de Pediatría y Cirugía Infantil Campus Occidente, Universidad de Chile. Santiago de Chile.

anterno de Medicina, Campus Occidente, Escuela de Medicina, Universidad de Chile.

Correspondencia a: Dr. Hayo Breinbauer K. Walter Scott 1439, Vitacura, Santiago. Teléfono celular: 8-2284576 - Teléfono fijo: 4948861. E mail: hayo@vtr.net 
"C uidador" se define como la persona, habitualmente un familiar directo, que convive y mantiene la responsabilidad de proveer recursos que un paciente, incapaz de autosustentarse, necesita"1. En la Encuesta Nacional de Salud de 2004 se registraron 917.939 chilenos (5,7\% de la población) como discapacitados moderados/severos, necesitados de apoyo en actividades de la vida diaria ${ }^{2}$. El $68,71 \%$ de ellos es cuidado por familiares directos.

Los adultos mayores (AM) con múltiples patologías o afectados por cuadros demenciales, hacen de éste un fenómeno creciente en nuestra población en vías de envejecimiento ${ }^{3}$. El 35,1\% de los discapacitados son AM (grupo que aumenta $0,15 \%$ anualmente). Se estima que $4 \%-7 \%$ de los AM en Chile (93.500 personas) son dependientes severos o postrados 4 .

En estos pacientes, el apoyo y características de sus familiares-cuidadores son los principales predictores pronósticos en cuanto a severidad sintomática y repercusiones sociales de sus cua$\operatorname{dros}^{5,6}$.

Asumir el rol de cuidador no es inocuo. Es posible desarrollar un fenómeno de "sobrecarga" por la tarea asumida, conjugando diversas variables: 1) Desatención de la propia salud, proyecto vital y vida social; 2) Deterioro familiar, relacionadas a dinámicas culposas, rabiosas y manipulatorias; 3) Ansiedad o frustración por falta de preparación y conocimiento técnico en el cuidado de pacientes dependientes 7,8 .

Una mayor sobrecarga deteriora la salud mental, social y física del cuidador, presentando con mayor frecuencia trastornos ansioso-depresi$\operatorname{vos}^{9,10}$, mayor aislamiento social, empeoramiento de la situación económica familiar ${ }^{11,12}$, mayor morbilidad general ${ }^{13}$ e incluso mayor mortalidad, que en población comparable no sobrecargada ${ }^{14}$. Además, el cuidador tiende a no buscar ayuda médica, postergándose y priorizando los problemas de su familiar dependiente, manteniendo la mayoría de sus propias patologías sin diagnosticar, tornándose casi "invisible" para el sistema de salud $^{15}$. Esto hace necesario evaluar esta situación en nuestro país.

Numerosos instrumentos intentan objetivar la sobrecarga del cuidador: "índice global de carga"; "entrevista de sobrecarga del cuidador"; "cuestionario de sentido de competencia"16. Sin embargo, estas herramientas evalúan sólo algunas dimensiones de este fenómeno, que incluye calidad de vida, capacidad de autocuidado, red de apoyo social y competencias para afrontar problemas conductuales y clínicos del paciente cuidado ${ }^{17}$.

La "Escala de sobrecarga del cuidador de Zarit" (EZ) ${ }^{18}$ logra aunar consistentemente todas estas dimensiones. Siendo la más utilizada a nivel internacional, ha sido validada en diversos idiomas incluyendo español ${ }^{19-21}$. EZ (Anexo 1) consta de 22 preguntas tipo Likert de 5 opciones (1-5 puntos), cuyos resultados se suman en un puntaje total (22-110 puntos). Este resultado clasifica al cuidador en: "ausencia de sobrecarga" ( $\leq 46)$, "sobrecarga ligera" (47-55) o "sobrecarga intensa" $(\geq 56)$. "Sobrecarga ligera" representa un factor de riesgo para generar "sobrecarga intensa". Esta última se asocia a mayor morbimortalidad médica, psiquiátrica y social del cuidador 22,23 .

Esta herramienta presenta gran confiabilidad inter-observador (coeficiente de correlación intraclase (CCI) 0,71 en su estudio original ${ }^{18}$, y $0,71-0,85$ en validaciones internacionales ${ }^{24-27}$ ), consistencia interna (alfa de Cronbach 0,91 en estudio original, y 0,85-0,93 en varios países $\left.{ }^{18,20,24-26}\right)$. Ha demostrado también gran validez de apariencia, contenido y constructo en diferentes adaptaciones lingüísticas ${ }^{26,28,29}$. En validez de criterio, EZ muestra alta correlación con instrumentos afines $(r=0,71$ con Índice Global de Carga; $r=0,41$ con Inventario Breve de Síntomas ${ }^{29}$ ). También se relaciona estrechamente con detección de otras patologías, principalmente en salud mental. Se estima $20,7 \%$ de trastornos mentales en cuidadores, siendo EZ capaz de discriminar distrés psíquico con una sensibilidad de $93 \%$ y especificidad de $80 \% 1$.

Más aún, el análisis factorial de las dimensiones que incorpora EZ, permite caracterizar dinámicas socioculturales de la población sobre la que se aplica, siendo útil en la programación de intervenciones ${ }^{23,24}$.

$\mathrm{Su}$ principal inconveniente es su extensión, por lo que recientemente se validó en España una forma abreviada, la "Escala de Zarit abreviada para cuidados paliativos" (EZA) ${ }^{30}$. Consta de sólo 7 ítems de la EZ original (ver Anexo 1). Cada ítem adopta 1-5 puntos, sumando entre 7 y 35 puntos. No distingue "sobrecarga ligera" de "sin sobrecarga", residiendo su utilidad en determinar "sobrecarga intensa". En su validación española obtuvo 


\section{Anexo 1. Escala de sobrecarga del cuidador de Z arit, versiones original (todos los ítems) y abreviada (ítems en gris)}

\begin{tabular}{|c|c|c|c|c|c|c|}
\hline \multirow{2}{*}{\multicolumn{2}{|c|}{ Puntuación para cada respuesta* }} & Nunca & $\begin{array}{l}\text { Rara } \\
\text { vez }\end{array}$ & $\begin{array}{c}\text { Algunas } \\
\text { veces }\end{array}$ & $\begin{array}{c}\text { Bastantes } \\
\text { veces } \\
4\end{array}$ & $\begin{array}{c}\text { Casi } \\
\text { siempre }\end{array}$ \\
\hline & & & & & & \\
\hline 1 & ¿Piensa que su familiar pide más ayuda de la que realmente necesita? & & & & & \\
\hline 2 & $\begin{array}{l}\text { ¿Piensa que debido al tiempo que dedica a su familiar no tiene } \\
\text { suficiente tiempo para usted? }\end{array}$ & & & & & \\
\hline 3 & $\begin{array}{l}\text { Se siente agobiado por intentar compatibilizar el cuidado de su } \\
\text { familiar con otras responsabilidades (trabajo, familia)? }\end{array}$ & & & & & \\
\hline 4 & ¿Siente vergüenza por la conducta de su familiar? & & & & & \\
\hline 5 & ¿Se siente enfadado cuando está cerca de su familiar? & & & & & \\
\hline 6 & $\begin{array}{l}\text { ¿Piensa que el cuidar de su familiar afecta negativamente la } \\
\text { relación que Ud. tiene con otros miembros de su familia? }\end{array}$ & & & & & \\
\hline 7 & ¿Tiene miedo por el futuro de su familia? & & & & & \\
\hline 8 & ¿Piensa que su familiar depende de usted? & & & & & \\
\hline 9 & $\begin{array}{l}\text { ¿Piensa que su salud ha empeorado debido a tener que cuidar } \\
\text { a su familiar? }\end{array}$ & & & & & \\
\hline 10 & ¿Se siente tenso cuando está cerca de su familiar? & & & & & \\
\hline 11 & $\begin{array}{l}\text { ¿Piensa que no tiene tanta intimidad como le gustaría debido a } \\
\text { tener que cuidar de su familia? }\end{array}$ & & & & & \\
\hline 12 & $\begin{array}{l}\text { Siente que su vida social se ha visto afectada negativamente por } \\
\text { tener que cuidar de su familiar? }\end{array}$ & & & & & \\
\hline 13 & $\begin{array}{l}\text { ¿Se siente incómodo por distanciarse de sus amistades debido a } \\
\text { tener que cuidar de su familiar? }\end{array}$ & & & & & \\
\hline 14 & $\begin{array}{l}\text { ¿Piensa que su familiar le considera a usted la única persona que } \\
\text { le puede cuidar? }\end{array}$ & & & & & \\
\hline 15 & $\begin{array}{l}\text { ¿Piensa que no tiene suficientes ingresos económicos para los } \\
\text { gastos de cuidar a su familiar, además de sus otros gastos? }\end{array}$ & & & & & \\
\hline 16 & $\begin{array}{l}\text { ¿Piensa que no será capaz de cuidar a su familiar por mucho } \\
\text { más tiempo? }\end{array}$ & & & & & \\
\hline 17 & $\begin{array}{l}\text { ¿Siente que ha perdido el control de su vida desde que comenzó } \\
\text { la enfermedad de su familiar? }\end{array}$ & & & & & \\
\hline 18 & ¿Desearía poder dejar el cuidado de su familiar a otra persona? & & & & & \\
\hline 19 & ¿Se siente indeciso sobre qué hacer con su familiar? & & & & & \\
\hline 20 & ¿Piensa que debería hacer más por su familiar? & & & & & \\
\hline 21 & ¿Piensa que podría cuidar mejor a su familiar? & & & & & \\
\hline & $\begin{array}{l}\text { Globalmente ¿Qué grado de "carga" experimenta por el hecho de } \\
\text { cuidar a su familiar? }\end{array}$ & & & & & \\
\hline
\end{tabular}

* Escala de Zarit (Todos los ítems): Cada respuesta obtiene una puntuación de 1 a 5. Luego se suma el puntaje obteniendo un resultado entre 22 y 110 puntos. Este resultado clasifica al cuidador en: "ausencia de sobrecarga" ( $\leq 46)$, "sobrecarga ligera" (47-55) y "sobrecarga intensa" ( 256$)$. El estado de sobrecarga ligera se reconoce como un riesgo para generar sobrecarga intensa. El estado de sobrecarga intensa se asocia a mayor morbimortalidad del cuidador.

** Escala de Zarit Abreviada (sólo los 7 ítems marcados en gris). Cada respuesta obtiene una puntuación de 1 a 5. Luego se suma el puntaje obteniendo un resultado entre 7 y 35 puntos. Este resultado clasifica al cuidador en: "ausencia de sobrecarga" ( $\leq 16)$ y "sobrecarga intensa" ( $\geq 17)$. El estado de sobrecarga intensa se asocia a mayor morbimortalidad del cuidador. 
$100 \%$ de sensibilidad, 90,5\% de especificidad, $95,5 \%$ de valor predictivo positivo (VPP) y 100\% valor predictivo negativo (VPN) frente al diagnóstico de sobrecarga intensa (según EZ original), convirtiéndose en una excelente herramienta de pesquisa $^{30,31}$. Se sigue recomendando la versión original de 22 preguntas para estudios comparativos, finos y con análisis de factores involucrados. Se plantea entonces el uso de EZA como instrumento de pesquisa y EZ como herramienta de estudio.

EZ sería también sensible al cambio. En estudios multicéntricos, controlados y aleatorios, diversos programas (basados en terapia ocupacional 0 cognitivo-conductual de enfoque comunitario) lograron disminuir significativamente niveles de sobrecarga, tanto en términos de EZ, como de calidad de vida, disminuyendo significativamente el riesgo de morbilidad física o mental asociada ${ }^{32-34}$.

Aunque EZ ya ha sido utilizada en algunos estudios recientes en Chile ${ }^{3,35}$, nunca ha sido formalmente validada. En un estudio cuantitativo se estimó que $28,1 \%$ de los cuidadores de $\mathrm{AM}$ en Santiago presentarían sobrecarga intensa ${ }^{3}$. La implementación de intervenciones gubernamenta$\mathrm{les}^{4}$, aunque dificultosa e incompleta, ya ha generado mejoras en los cuidadores, en términos de puntaje EZ y calidad de vida ${ }^{3,36}$. Más aún, considerar al paciente dependiente y su cuidador como una diada interrelacionada, necesitada de una atención integral, no forma aún parte de la práctica clínica habitual en nuestro medio 3,22,37.

Así, cobra relevancia contar con instrumentos validados en Chile, que permitan objetivar (y por ende intervenir dirigidamente) la sobrecarga de cuidadores de pacientes dependientes.

El objetivo de este estudio es contribuir a validar en nuestro medio la escala de sobrecarga del cuidador de Zarit, tanto en su versión original como abreviada.

\section{Metodología}

Estudio de validación psicométrica, realizado entre marzo y junio de 2008.

Tamaño muestral. Utilizando transformación de Fisher, en base al mínimo valor de correlación descrito para EZ $(0,71)^{18}$, considerando variación de 0,2 en dicha correlación, seguridad de 95\% (bilateral) y poder estadístico de $80 \%$, se determinó un tamaño muestral de 28 cuidadores para determinar correlaciones entre variables ${ }^{38}$.

Serían necesarios 220 pacientes (10 sujetos por ítem del instrumento, por criterios de factibilidad ${ }^{38}$ ) para realizar análisis factorial. Aunque éste permitiría analizar las dimensiones involucradas (apoyando validez de constructo) por motivos prácticos se decidió dejar para un estudio posterior.

Sujetos. Criterio de inclusión: presencia de diada cuidador-paciente, que cumpliese la definición descrita en la introducción, considerando como dependientes a pacientes con Katz $\geq 2^{30,39}$.

La muestra consistió en 22 individuos pertenecientes al programa "VIDA" de atención domiciliaria a pacientes dependientes o postrados del Centro de Salud San Manuel de Melipilla y 10 individuos no pertenecientes a dicho programa, asistentes al mismo centro de salud. Los cuidadores del programa "VIDA" representan una población cautiva del consultorio (permitiendo su seguimiento) y desde comienzos de 2008 se les aplica regularmente EZ dentro del programa. El estudio contó con la aprobación y apoyo de la Corporación de Educación y Salud de Melipilla (supervisor del centro de salud en cuestión). Todos los cuestionarios aplicados en este estudio formaban parte de las evaluaciones rutinarias propias del programa.

Instrumentos aplicados. Previo consentimiento informado, se aplicaron: 1) Escala de sobrecarga del cuidador de Zarit en español (EZ) ${ }^{20}$. Se implementó sin traducciones o cambios, considerando como experiencia piloto su reiterada aplicación previa dentro del programa "VIDA" sin dificultades de comprensión (además, el mismo formato ha sido utilizado en Chile sin contratiempos $\left.^{3,35,36}\right)$; 2) EZ abreviada para cuidados paliativos en español (EZA) ${ }^{30,31}$. Al consistir en una selección de los ítems de EZ, tampoco fue necesario realizar traducciones. 3) Indicador único, global y subjetivo para sensación de sobrecarga. Ítem tipo Lickert con rango 1-5 puntos. Su correlación con EZ y EZA se utilizó como aporte a la validez de constructo; 4) Encuesta de depresión CIE- $10^{40}$. Su correlación con EZ y EZA se utilizó como aporte a la validez de constructo. 
Se registraron edad y sexo del cuidador y paciente, años cumpliendo función de cuidador, número de salidas mensuales fuera del hogar y realización de otras actividades fuera del cuidado, para comparar los pacientes del programa "VIDA" con los otros 10 individuos, sin encontrarse diferencias significativas, permitiendo su análisis como un solo grupo.

Los instrumentos fueron aplicados en 3 oportunidades. Dos veces por observadores distintos al inicio del estudio (explorando confiabilidad interobservador), y una tercera vez 4 meses después por uno de los equipos iniciales (explorando confiabilidad test-retest). La segunda y tercera aplicación sólo consideró a los 22 pacientes del programa VIDA.

Determinación de validez. 1) La validez de apariencia y contenido han sido extensamente revisadas en numerosos estudios internacionales y no realizamos mayores aportes en este estudio; 2) para validez de constructo, se correlacionaron (Pearson) ambas escalas con la escala de depresión, y con indicador único de sobrecarga. El marco teórico supone que de incrementarse la sobrecarga detectada por EZ o EZA, aumentarían tanto la frecuencia de depresión, como la impresión subjetiva de sobrecarga. Se consideró una asociación fuerte con coeficiente de correlación $\geq 0,5^{38,41}$; 3) para validez por criterio externo de EZA, utilizamos los resultados de "sobrecarga intensa" en EZ como gold standard ${ }^{30}$, buscando determinar si EZA es capaz, por sí sola, de discriminar sobrecarga intensa. Se consideró este procedimiento como válido, dados los altos niveles de validez y confiabilidad que caracterizan a EZ en los estudios internacionales descritos, y en los resultados presentados en este estudio. Se realizó correlación de Pearson entre las escalas, análisis de curvas ROC, y cálculos de sensibilidad-especificidad $38,41,42$.

Determinación de confiabilidad para cada escala. 1) Se determinó consistencia interna con alfa de Cronbach. Se consideraron valores $\geq 0,75$ como alta consistencia; 2) la confiabilidad por estabilidad se evaluó aplicando índice kappa en testretest, para cada ítem comparando dos aplicaciones con 4 meses de diferencia. Se consi- deraron valores $\geq 0,6$ como acuerdo sustanciales y $\geq 0,8$ como acuerdo casi perfecto; 3) la confiabilidad interobservador se midió con CCI entre dos aplicaciones de la escala por observadores distintos. En su interpretación, se consideraron rangos equivalentes al kappa.

Sensibilidad al cambio. No fue evaluada en este estudio.

Determinación de utilidad. Se recogió la experiencia de los 8 entrevistadores, comentando duración de aplicación de los instrumentos, necesidad de entrenamiento y facilidad de calificación.

Para el análisis estadístico se utilizó software SPSS 16.0, manteniendo seguridad de 95\%.

\section{RESULTADOS}

La edad promedio de los cuidadores fue 55,6 años, 89,3\% eran mujeres, 89,3\% eran hijas de quienes cuidaban y llevaban en promedio 10,1 años ejerciendo como cuidadoras. El 77,3\% sólo se dedicaban a esta actividad y salían sólo 4,4 veces al mes fuera de su hogar. En EZ 52,9\% mostró sobrecarga intensa, $17,6 \%$ sobrecarga ligera y $29,4 \%$ no presentó sobrecarga.

La Tabla 1 muestra los resultados obtenidos para validez y confiabilidad en EZ y EZA.

Validez de constructo. Ambos instrumentos mostraron correlaciones fuertes tanto con el indicador único de sobrecarga, como con depresión $(r=0,51-0,7 ; p<0,05)$.

Validez de criterio. EZA se correlacionó de forma casi perfecta con EZ ( $r=0,92 ; p<0,001)$.

Sensibilidad y especificidad EZA. La Figura 1 ilustra la curva ROC para EZA controlada por el diagnóstico de sobrecarga intensa en EZ. El área bajo su curva fue 0,957 ( $p<0,0001$ ). Su análisis permitió determinar 17 puntos (dentro del rango 7-35) como el mínimo punto de corte necesario para detectar sobrecarga intensa con $100 \%$ de sensibilidad. Este es el mismo punto de corte descrito en su validación española ${ }^{30}$. Con este corte obtuvo además $77,7 \%$ de especificidad, VPP de $86,6 \%$ y VPN de $100 \%$ (Tablas 2 y 3 ). 
Tabla 1. Resultados de pruebas de validez y confiabilidad para ambos instrumentos

\begin{tabular}{|c|c|c|c|c|c|}
\hline & Aspecto evaluado & Prueba Estadística & (n) & Escala de Zarit (EZ) & $\begin{array}{l}\text { Escala de Zarit } \\
\text { abreviada (EZA) }\end{array}$ \\
\hline \multirow{4}{*}{$\frac{8}{3}$} & Validez de constructo & Correlación de Pearson & 32 & 0,67 & 0,51 \\
\hline & & $\begin{array}{l}\text { con indicador único } \\
\text { de sobrecarga }{ }^{b}\end{array}$ & & $\mathrm{p}<0,05$ & $\mathrm{p}<0,001$ \\
\hline & & Correlación de Pearson & 32 & 0,7 & 0,67 \\
\hline & $\begin{array}{c}\text { Validez de criterio } \\
\text { externo }\end{array}$ & $\begin{array}{l}\text { Correlación entre } \\
\text { puntuación EZA con EZ } \\
(\text { Gold-Standard) })^{b}\end{array}$ & 32 & - & $\begin{array}{c}0,92 \\
p<0,001\end{array}$ \\
\hline \multirow{3}{*}{$\begin{array}{l}\frac{7}{0} \\
\frac{7}{0} \\
\frac{0}{0} \\
0\end{array}$} & Consistencia interna & Alfa de Cronbach ${ }^{\mathrm{a}}$ & 32 & 0,87 & 0,84 \\
\hline & $\begin{array}{c}\text { Confiabilidad } \\
\text { interobservador }\end{array}$ & $\begin{array}{l}\text { Coeficiente correlación } \\
\text { intraclase }(\mathrm{CCI})^{\mathrm{C}}\end{array}$ & 22 & $\begin{array}{c}0,86 \\
\left(0,81-0,91^{d}\right)\end{array}$ & $\begin{array}{c}0,81 \\
\left(0,76-0,87^{d}\right)\end{array}$ \\
\hline & $\begin{array}{l}\text { Confiabilidad en } \\
\text { estabilidad }\end{array}$ & Kappa en Test - Retest ${ }^{\mathrm{C}}$ & 22 & $\begin{array}{c}0,91 \\
\left(0,68-0,99^{\mathrm{e}}\right)\end{array}$ & $\begin{array}{c}0,93 \\
\left(0,74-0,99^{f}\right)\end{array}$ \\
\hline
\end{tabular}

a Valores en Alpha de Cronbach mayores a 0,75 son considerados como alta consistencia interna.

b Valores en Pearson mayores a 0,5 con p <0,05 traducen una correlación fuerte entre ambas variables.

${ }^{c}$ Valores en CCI y Kappa mayores a 0,6 son considerados como acuerdo sustancial entre dos evaluaciones, y mayores a 0,8 como acuerdo casi perfecto entre dos evaluaciones.

d Intervalo de confianza de 95\%-

e Rango de Kappa obtenido al evaluar independientemente cada uno de los 22 indicadores.

${ }^{\mathrm{f}}$ Rango de Kappa obtenido al evaluar independientemente cada uno de los 7 indicadores.

Tabla 2. C ruce de resultados positivos para sobrecarga intensa entre ambos instrumentos

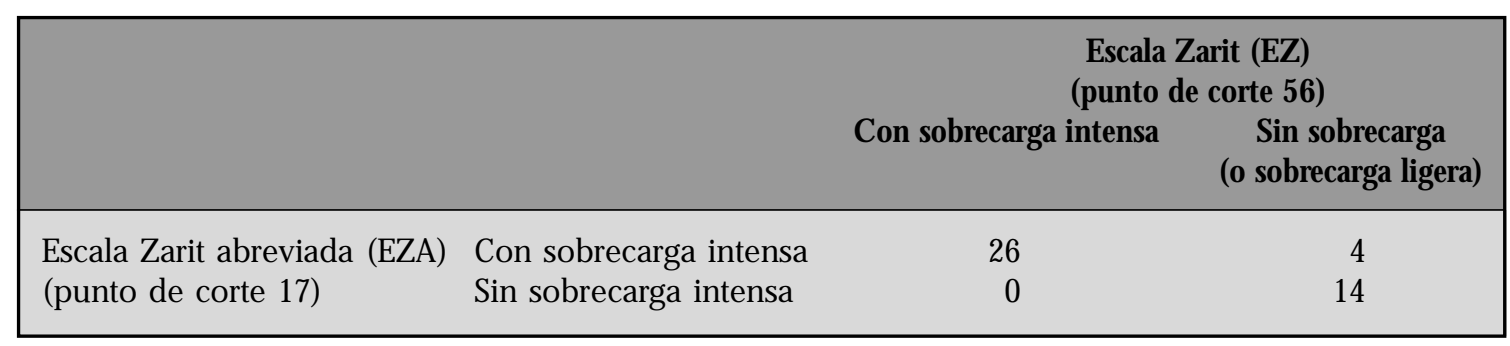

Tabla 3. Características de la escala de Zarit abreviada (versión diseñada originalmente para cuidados paliativos) en nuestro medio

\begin{tabular}{|lcc|}
\hline & Valor & Intervalo de confianza con 95\% de seguridad \\
\hline Sensibilidad & $100 \%$ & $97,2-100$ \\
Especificidad & $77,7 \%$ & $73,3-81,9$ \\
Valor predictivo positivo & $86,6 \%$ & $84,6-88,6$ \\
Valor predictivo negativo & $100 \%$ & $96,6-100$ \\
\hline
\end{tabular}


Figura 1. Curvas ROC para escala de Zarit abreviada (EZA), en base a categoría "Sobrecarga Inten$\mathrm{sa}^{\prime}$ en escala de Zarit original (EZ).

Consistencia interna. Ambos instrumentos alcanzaron altos niveles en alfa de Cronbach.

Confiabilidad interobservador. EZ mostró acuerdo casi perfecto entre observadores, con valores sobre 0,81 en el margen inferior del intervalo de confianza del CCI. EZA mostró rangos sólo un poco menores y siempre con nivel de acuerdo sustancial entre observadores.

Confiabilidad de estabilidad. Ambos instrumentos mostraron altos índices kappa comparando el puntaje total en ambas aplicaciones. Independientemente, todos los ítems mantuvieron un acuerdo test-retes al menos sustancial, obteniendo el más discordante un kappa de 0,68.

Utilidad. Los encuestadores consideraron ambos instrumento de fácil aplicación. Todos los ítems y la forma de contestar fueron comprendidos rápidamente. Leer detenidamente la encuesta una vez fue suficiente entrenamiento para sentirse preparados a implementarla. Aplicar EZ no demora más de $8 \mathrm{~min}$ y EZA menos de 3 min. Calificar ambas escalas fue simple, al sumarse los indicadores de forma lineal.

\section{DisCUSIONES}

Tanto EZ como EZA mostraron altos niveles de validez de constructo en relación a dos dimensiones

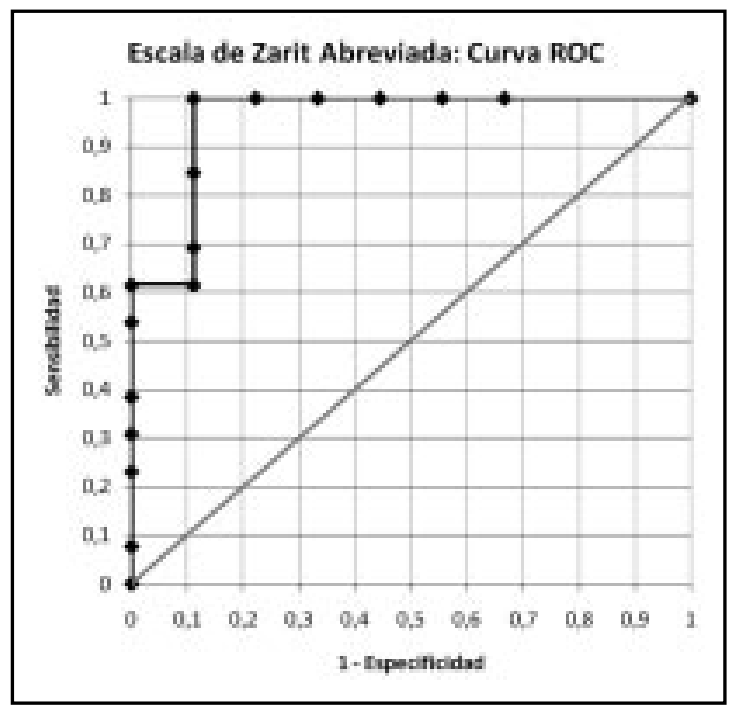

del instrumento (indicador único subjetivo y depresión). Ambas escalas evidenciaron además excelentes valores de consistencia intema, confiabilidad interobservador, y confiabilidad por estabilidad test-retest.

EZA se correlacionó fuertemente con EZ, apoyando la validez por criterio externo de EZA. Considerando además la gran área bajo curva ROC, pareciera que EZA evalúan el mismo fenómeno que EZ, logrando discernir presencia de sobrecarga intensa en cuidadores.

Destaca la potencial utilidad que reviste EZA, como método de pesquisa en atención primaria para identificar a cuidadores con sobrecarga intensa, considerando una sensibilidad y VPN de 100\%, además de una alta especificidad y VPP.

Sin embargo, debemos considerar algunos sesgos en esta validación. 1) Tamaño muestral. Aunque apropiado para determinar las comelaciones implementadas, serían necesarios 220 cuidadores para realizar un análisis factorial. Este permitiría una validación de constructo más amplia, además de explorar dinámicas subyacentes en la realidad sociocultural de los cuidadores ${ }^{23}$. 2) No se determinó sensibilidad al cambio. Saber si el instrumento es capaz de registrar el impacto de intervenciones es crucial para elaborar programas de apoyo. Realizarlo requerińa un estudio controlado de al menos 6 meses. Sin embargo, se describe una adecuada sensibilidad al cambio en la EZ, siendo utilizada como medida de control en importantes estu$\operatorname{dios}^{32,34}$. 3) Ruralidad de la muestra. La validez y 
confiabilidad evidenciadas en este estudio no pueden ser inmediatamente extrapoladas a un medio urbano. Un mayor acceso a recursos en un sector urbano, pudiesen mitigar los efectos de la sobrecarga (un estudio en Santiago estimó casi la mitad de sobrecarga respecto a la registrada en este estudio ${ }^{3}$ ), alterando principalmente la validez de constructo sustentada en la comelación de los instrumentos con sensación subjetiva de sobrecarga y depresión.

Por otro lado, los resultados en este estudio son semejantes a los descritos en numerosos estudios internacionales de validez y confiabilidad, especialmente para $\mathrm{EZ}^{5,6,15,20,26,29,31,43}$, realizados en medios urbanos y rurales. Bajo esta consideración, los sesgos descritos tendrían bajo impacto sobre la validez y confiabilidad de estos instrumentos en Chile.

En suma, se considera que ambos instrumentos pudiesen utilizarse válidamente en Chile, para objetivar sobrecarga y guiar procesos de intervención en diadas cuidador-paciente dependiente.

\section{CONCLUSIONES}

Este estudio contribuye a validar la Encuesta de Sobrecarga del Cuidador de Zarit, en sus versiones

\section{REFERENCIAS}

1. Martín M, Baliesteros J, Ibarra N, Loizaga C, Serrano B, LARUMBE MJ ET AL. Sobrecarga del cuidador de pacientes con enfermedad de Alzheimer y distrés psíquico. Una asociación relegada en la valoración de las demencias. Actas Esp Psiquiatr 2002; 30: 201-6.

2. Fondo NaCional DE IA Discapacidad, INE. Estudio Nacional de la Discapacidad ENDISC 2004. Santiago, Chile 2004. [citado el 21 de octubre de 2008] Disponible en: www.fonadis.cl.

3. Albala C, Sánchez H, Bustos C, Fuentes A, lera L. Situación de los cuidadores domiciliarios de los adultos mayores dependientes con pensión asistencial: INTA, Universidad de Chile; 2007.

4. Ministerio Secretaría General de ta Presidencia. Cumplimiento Plan 100 días, 36 Medidas, Gobiemo Presidenta Michelle Bachelet, Junio 2006. [citado el 21 de octubre de 2008] Disponible en: www.minsegpres.gob.cl

5. Argimon JM, Limon E, Viшa J, Cabezas C. Healthrelated quality-of-life of care-givers as predictor of nursing-home placement of patients with dementia. Alzheimer Dis Assoc Disord 2005; 19: 41-4. original y abreviada para cuidados paliativos. Recomendamos ambos instrumentos como útiles y válidos, principalmente en el contexto de atención primaria, para la detección de sobrecarga en cuidadores de pacientes dependientes y su eventual intervención.

Con su brevedad y $100 \%$ de sensibilidad, EZA representa un excelente método de pesquisa para detectar cuidadores que presenten sobrecarga intensa, condición asociada a un aumento de morbimortalidad ${ }^{30,31}$. La EZ por su parte, en sus 22 preguntas originales, se mantiene como un instrumento de estudio y seguimiento más fino, que permite guiar y controlar programas de intervención probados en el extranjero, como grupos de apoyo, terapia ocupacional de enfoque comunitario, terapia cognitiva conductual y entrega de herramientas de manejo de pacientes dependientes ${ }^{32-34}$.

\section{Agradecimientos}

Queremos agradecer a la directora del Departamento de Salud de la Corporación de Educación y Salud de Melipilla, Dra. Nelly Madrid, a los funcionarios del Centro de Salud San Manuel en Melipilla, y a los participantes del intemado rural del Campus Occidente de la Escuela de Medicina de la Universidad de Chile, del período abril-julio de 2008, sin los cuales este estudio no habría sido posible.

6. Brodaty H, Green A, Koshera A. Meta-analysis of pshychosocial interventions for caregivers of people with dementia. J Am Geriatr Soc 2003; 51: 657-64.

7. HALEY WE. The family caregiver's role in Alzheimer's disease. Neurology 1997; 48: 25-9.

8. WhitLatch CJ, ZaRT SH. Efficacy of interventionts with caregivers: a reanalysis. Gerontologist 1991; 31: 9-14.

9. Thompson C, Briggs M. Support for carer of people with Alzheimer's type dementia. Cochrane Databes Syst Rev 2000; 2: CD000454.

10. Marriot A, Donaddson C, Terrier N, Burns A. Effectiveness of cognitive-behavioural family intervention in reducing the burden of care in carers of patients with Alzheimer's disease. Br J Psychiatry 2000; 176: 557-62.

11. Mittelman MS, Ferris SH, Shulman E, Steinber G, Levin B. A family intervention to delay nursing home placement of patients with Alzheimer disease. A randomized controlled trial. JAMA 1996; 276: 1725-31.

12. Wisniewski S, Belle SH, Coon DW, Marcus SM, Ory MG, BuRgio LD ET AL. The Resources for Enhancing Alzheimer's Caregiver Health (REACH): project design and baseline characteristics. Psychol Aging 2003; 18: 375-84. 
13. López O, LOREnzo A. Morbilidad en cuidadores de pacientes confinados en su domicilio. Aten Primaria 1999; 24: 404-10.

14. Schulz R, BEACH S. Caregiving as a risk factor for mortality. The caregiver health effects study. JAMA 1999; 282: 2215-9.

15. Hepburn KW, Tornatore J, Center B, Ostwald SW. Dementia family caregiver training: affecting beliefs about caregiving and caregiver outcomes. J Am Geriatr Soc 2001; 49: 450-7.

16. Schulz R, O’Brien A, Czaja S, Ory M, Norris R, Martire LM ET AL. Dementia caregiver intervention research: in search of clinical significance. Gerontologist 2002; 42: 589-602.

17. Schulz R, Mendelsohn AB, Haley WE, Mahoney D, ALEN RS, Zhang S, ET AL. Resources for Enhancing Alzheimer's Caregiver Health Investigators. End-oflife care and effects of bereavement on family caregivers of persons with dementia. N Engl J Med 2003; 349: 1936-42.

18. Zarit SH, ReEver KE, Bach-Peterson J. Relatives of the impaired elderly: correlates of feelings of burden. Gerontologist 1980; 20: 649-55.

19. Wisniewsis S, Belue S, Coon D, Marcus S, Ory M, Burgio L ET AL. The Resources for Enhancing Alzheimer's Caregiver Health (REACH): project design and baseline characteristics. Psychol Aging 2003; 18: 375-84.

20. Martín M, Salvadó I, Nadal S, Miji LC, Rico JM, Lanz P. Adaptación para nuestro medio de la Escala de Sobrecarga del Cuidador (Caregiver Burden Interview) de Zarit. Rev Gerontol 1996; 6: 338-46.

21. Ankri J, Andrieu S, Beaufils B, Grand A, Henrard J. Beyond the global score of the Zarit Burden Interview: useful dimensions for clinicians. Int J Geriatr Psychiatry 2005; 20: 254-60.

22. ZARIT SH, ORR NK, ZARIT JM. The hidden victims of Alzheimer's disease: families inder stress. New York: New York University Press. 1985.

23. Ankri J, Andrieu S, Beaufils B, Grand A, Henrard JC. Beyond the global score of the Zarit Burden Interview: useful dimensions for clinicians. Int J Geriatr Psychiatry 2005; 20: 254-60.

24. LeE YR, Sung KT. Cultural influences on caregiving burden: cases of Korean and Americans. Int J Aging Hum Dev 1998; 46: 125-41.

25. Demers A, LavoIe JP. Effect of support groups on familiy caregiver to the frail elderly. Can J Aging 1996; 15: 129-44.

26. Arai $Y$, Kudo K, Hosokawa T, Washio M, Miura $H$, Hisamichi S. Reliability and validity of the Japanese version of the Zarit caregiver buden interview. Psychiatry Clin Neurosci 1997; 51: 281-7.

27. Taub A, Andreoli SB, Bertolucci PH. Dementia caregiver burden: reliability of the Brazilian version of the Zarit caregiver burden interview. Cad Saúde Pública 2004; 20: 372-6.
28. ZARIT SH, ZaRTT JM. The Memory and behavior problem checklist and the burden interview: Technocal Report, Pennsylvania State University; 1983.

29. Hérbert R, Bravo G, PrÉviLe M. Reliability, validity and reference values of the Zarit Burden Interview for assesing infomral caregivers of communiti-dwelling older persons with dementia. Can J Aging 2000; 19: 194-507.

30. Regueiro AA, Pérez-Vásquez A, Gómara S, Ferreriro M. Escala de Zarit reducida para la sobrecarga del cuidador en atención primaria. Aten Primaria 2007; 39: 185-8.

31. Gort AM, March J, Gómez X, de Miguel M, Mazarico S, BALESTÉ J. Short Zarit scale in palliative care. Med Clin (Barc) 2005; 124: 651-3.

32. Belle S, Burgio L, Burns R, Coon D, Czaja S, GaLAGHER-Thompson D ET AL. Enhancing the Quality of life of Dementia Caregivers from Different Ethnic or Racial Groups. Ann Intern Med 2006; 145: 727-38.

33. Nichols LO, Chang C, Lummus A, Burns R, MarindaleAdams J, Graney MJ, et aL. The Cost-Effectiveness of a Behavior Intervention with Caregivers of Patients with Alzheimers's Disease. J Am Geriatr Soc 2008; 56: 413-20.

34. Graff MJL, Vernooij-Dassen MJM, Thijssen M, DekKer J, Hoefnagels WHL, Olde-RikKerT MGM. Community based occupational therapy for patients with dementia and their care givers: randomised controlled trial. BMJ 2006 Dec 9; 333(7580): 1196. Epub 2006 Nov 17. Disponible en www.bmj.com.

35. Gutiérrez-Maldonado J, Caqueo-Unízar A, Kavanagh DJ. Burden of care and general health in families of patients with schizophrenia. Soc Psychiatry Psychiatr Epidemiol 2005; 40: 899-904.

36. Fondo Nacional de la Discapacidad. Cuidadoras y cuidadores de personas dependientes y enfoque de género en Chile. Santiago, Chile 2007. [citado el 21 de octubre de 2008]. Disponible en: www.fonadis.cl

37. Alonso A, Garrido A, Díaz A, Casquero R, Riera M. Profile and Burden of Care in Caregivers of Patients with Dementia included in the ALOIS Program. Aten Primaria 2004; 33: 61-8.

38. ARgimón JM, JimÉNEZ J. Métodos de Investigación Clínica y Epidemiológica. $2^{\mathrm{a}}$ ed. Madrid: Ediciones Harcourt, 2000.

39. Katz S, Акром CA. A measure of primary sociobiological functions. Int J Health Serv 1976; 6: 493-508.

40. Ministerio de Salud. Guía Clínica. Serie Guias Clínicas Minsal NXX, $1^{\mathrm{a}}$ edición. Santiago: Minsal, 2006.

41. Alarcón AM, Muñoz S. Medición en salud: Algunas consideraciones metodológicas. Rev Méd Chile 2008; 136: $125-30$.

42. SÁnchez R, EcheverRY J. Validación de Escalas de Medición en Salud. Rev Salud Pública 2004; 6: 302-18.

43. Gort A, Mingot M, Gómez X, Soler T, Torres G, SACRISTÁN O, ET AL. Use of Zarit scale for assesing caregiver burden and collapse in caregiving at home in dementias. Int J Geriatr Psychiatry 2007; 22: 957-62. 